\title{
Vídeos agregam valor ao trabalho do professor de ciências da saúde
}

Maria Isabel Timm - Centro Nacional de Supercomputação (CESUP)- Universidade

Federal do Rio Grande do Sul (UFRGS), beta@ cesup.ufrgs.br

Adriana Bos-Mikich , Departamento de Ciências Morfológicas, do Instituto de Ciências

Básicas da Saúde (ICBS), da UFRGS, adriana.bosmikich@gmail.com

Roberto Thaddeu, Departamento de Farmacologia do ICBS-UFRGS, farmaco@ufrgs.br

Ana Maria Ponzio de Azevedo, Departamento de Ciências Básicas da Saúde da

Universidade Federal de Ciências da Saúde (UFCSPA), anzevedo@ufcspa.edu.br

Paulo Augusto Cabral Jr. - UFRGS/TV - pazcabral@yahoo.com.br

Resumo: O trabalho apresenta exemplos de vídeos produzidos para o ensino médico. Justifica o uso de vídeos nesta área para apoiar a visualização de fenômenos cuja observação in loco depende de situações não reprodutíveis - como cirurgias ou dissecações - ou exigem alta capacidade de abstração dos alunos, no caso de fenômenos microscópicos, químicos, ou moleculares. Defende que os vídeos não induzem à passividade dos alunos, se forem produzidos de forma criativa e coerente, explorando os recursos da linguagem de vídeo associados ao potencial da tecnologia digital. Os exemplos também apresentam recursos de produção e edição, salientando-se a intenção didática ou pedagógica que gerou a escolha por um ou outro recurso. Sugere que vídeos educacionais contribuem para a aprendizagem dos alunos e agregam valor ao trabalho do professor, porque apóiam a representação e a exposição do conhecimento e podem constituir objeto de estudo, pesquisa e profissionalização de equipes de produção, em todas as instituiçõos que pretendem atualizar sua base tecnológica, segundo necessidades impostas pelo Séc. XXI.

Palavras-chave: vídeos educacionais, ensino médico, ensino de ciência e tecnologia, tecnologia educacional

\section{Videos add value to health sciences teachers' work}

Abstract: The paper relates examples of educational videos, produced for medical education. It defends the use of videos in this area, to support visualization of phenomena which happens on not reproducible situations - like surgeries or dissections - or demands students abstraction high skills, which is the case of microscopic, chemical, or molecular phenomena. It also argues that videos do not encourage passivity in students' behavior, if they are produced in a creative and professional way, exploiting video language resources, in association with digital technology potential. It also features examples of production and editing, stressing the didactical or pedagogical intention that generated the choice for one or another resource. It suggests that educational videos contribute to students' learning and add value to the work of the teacher, because support knowledge representation and exposure, and may be objects of study, research and professional teams of production, in all institutions wishing to upgrade their basic technology, according requirements imposed by the twenty-first century. 
Keywords: educational videos, medical education, teaching of science and technology, educational technology

\section{Introdução: representação e visualização de fenômenos como apoio à cognição}

O ensino das ciências médicas contemporâneo se depara hoje com a urgência de aproveitar os enormes recursos disponibilizados através da massificação das tecnologias audiovisuais informatizadas. Sugere-se neste trabalho que tais tecnologias, em especial os vídeos, dão importante suporte à visualização de fenômenos complexos dessas áreas, cuja observação in loco depende de situações não reprodutíveis - como cirurgias ou dissecações - ou de alta capacidade de abstração dos alunos, no caso de fenômenos microscópicos, químicos, ou moleculares. Nesse sentido, caracterizam-se como recursos importantes a serem integrados no cotidiano do trabalho docente, uma vez que agregam valor à capacidade representacional do professor. Ao contrário de uma visão disseminada nos debates didático-pedagógicos, os autores deste trabalho acreditam que os vídeos não são uma tecnologia obsoleta nas situações de ensino-aprendizagem do ensino médico ${ }^{1}$ e tampouco induzem à passividade de seus assistentes. Vão captar e manter a atenção e o interesse do aluno, principalmente se forem planejados para explorar de forma criativa e coerente a linguagem de vídeos, bem como os recursos da documentação e da representação de fenômenos, associados ao enorme potencial da tecnologia digital, seja para uso presencial, para apoiar o professor em sala de aula, ou à distância, através da Internet.

As áreas científicas e tecnológicas têm grande necessidade de acesso a conteúdos estruturados e extensos (Timm et al., 2004). O professor destas áreas não pode e não deve furtar-se de sistematizar o conhecimento já existente, e apresentá-lo (ou disponibilizá-lo em vídeo) de forma precisa e atraente. Com isto, estará realmente ajudando os alunos a compreender os fenômenos apresentados, sejam eles estáticos ou dinâmicos, macro ou microscópicos, teóricos ou práticos, e clínicos ou experimentais, no caso do ensino médico. Os vídeos podem dar suporte à construção endógena do conhecimento, pelo aluno, individualmente ou através das atividades em grupo, desde que haja uma conveniente sinergia entre os estímulos visuais e auditivos, integrados para ofertar a devida redundância à informação a ser processada, através da linguagem adequada desta mídia, reconhecida como tal pelo hábito cultural do cinema e da televisão. $\mathrm{O}$ uso consciente dos recursos audiovisuais deve contribuir para mobilizar a percepção e a atenção do aluno, destacando os itens mais relevantes (através de cor, movimento, legendas de apoio, edição conjunta com imagens ou vídeos anteriormente gravados, entre outros recursos), de forma a facilitar os processos de aprendizagem no que se refere à aquisição de conteúdos e sua consolidação como memória de longa duração, ao destacar para o aluno as partes mais fundamentais desse conteúdo.

Os vídeos - como recursos de representação e apoio à cognição e, portanto, como ferramenta pedagógica - podem/devem ser agregados a outros recursos multimediáticos, para o planejamento das atividades educacionais, associados a bancos de dados e de casos clínicos, ferramentas de discussão síncrona ou assíncrona (chats e fóruns), pesquisa e

\footnotetext{
${ }^{1} \mathrm{O}$ artigo refere-se ao ensino médico, mas os autores consideram que os vídeos dão suporte a todas as áreas do conhecimento, adaptando-se a necessidades, conteúdos e níveis desejados da atividade educacional.

V. 6 No 2, Dezembro, 2008
} 
interatividade, entre outros elementos de acesso à informação e apoio à comunicação e à interatividade (Schnaid et al., 2006). Mais do que adequar-se, em princípio, a uma ou outra intenção pedagógica, sugere-se que a tecnologia educacional - vídeos, no caso deste trabalho - precisa estar contextualizada e integrada a um conjunto flexível e variado de materiais e estratégias, em benefício da aprendizagem, vista como um fenômeno tanto biológico quanto cultural, e também do ensino, através da agregação de valor ao trabalho do professor do Século XXI.

\section{Exemplos de planejamento e de recursos de linguagem}

Serão apresentados exemplos de vídeos que utilizam recursos de produção e edição, salientando-se exatamente os detalhes audiovisuais e a intenção que gerou a escolha por um ou outro recurso, dentro do vasto repertório de possibilidades existentes na linguagem de produção de vídeos em geral. Deixam de ser apresentados neste trabalho tanto os aspectos técnicos relativos a tipos de equipamentos utilizados, bem como os aspectos relativos à observação do uso dos vídeos pelos alunos ${ }^{2}$, em função do objetivo proposto de salientar, para os professores da área médica, as possibilidades da linguagem dos vídeos como apoio à representação de seu próprio conhecimento e como instrumento eficiente para mediar as necessidades didático-pedagógicas em sala de aula. Deixa-se de fazer referência também aos aspectos de áudio, seja através da voz do professor sincronizada com as imagens e caixas de texto, seja através de sonoplastia, trilha sonora, vinhetas e outros recursos que podem contribuir para manter a atenção do aluno. Os exemplos serão apresentados na forma de duas tabelas, com colunas de imagens, descritas e analisadas ao lado, em termos do recurso utilizado e das intenções que geraram sua escolha na linguagem do vídeo. Na tabela 1 os exemplos fazem parte de uma série de três vídeos ${ }^{3}$ produzidos para estruturar os conteúdos da Neuro e Psicofarmacologia, direcionados a várias disciplinas, entre as quais as de Farmacologia Médica, do Departamento de Farmacologia do Instituto de Ciências Básicas da Saúde (ICBS), da Universidade Federal do Rio Grande do Sul (UFRGS).

Tabela 1 - Exemplos de Vídeo Educacional em Psicofarmacologia

\begin{tabular}{|c|l|}
\hline $\begin{array}{c}\text { Imagens tiradas de cenas dos vídeos de } \\
\text { aulas gravadas em estúdio, com } \\
\text { ilustrações em 3D }\end{array}$ & $\begin{array}{l}\text { Descrição e análise das imagens, suas } \\
\text { características em termos de linguagem } \\
\text { audio-visual e sua intenção didática, em } \\
\text { termos de representação do conteúdo. }\end{array}$ \\
\hline & Animação utilizada para que o aluno possa \\
\hline
\end{tabular}

\footnotetext{
${ }^{2}$ No caso da série de vídeos sobre psicofarmacologia, foi realizada pesquisa sobre aquisição e retenção de conteúdos, em comparação com aula presencial. Partiu-se de uma hipótese - baseada no senso comum - de que a aula gravada em vídeo deve ser menos eficiente do que a exposição presencial, dialogada com o professor, principalmente pela característica fixa dos conteúdos e por não possibilitar perguntas e esclarecimentos ao aluno. Essa dedução ainda que lógica, mostrou-se enganosa, e abriu um importante espaço para o debate sobre o uso de aulas expositivas gravadas, como parte de um planejamento de cursos presenciais, semi-presenciais e a distância, sem prejuízo de outros materiais interativos, nem de seu uso em atividades objetivistas ou construtivistas, dirigidas ou autônomas, individuais ou em grupo. (Cabral Jr. et al , 2005). O outro vídeo apresentado neste artigo, uma aula de Embriologia, está em fase de elaboração de metodologia de uso e avaliação.

${ }^{3}$ Os três títulos da série de vídeos são: Psicofármacos (ação no sistema nervoso central); Psicofarmacologia experimental (com o professor Roberto Thaddeu); e Hipnosedativos (com a professora Elaine Elisabetsky)
}

V. 6 No 2, Dezembro, 2008 


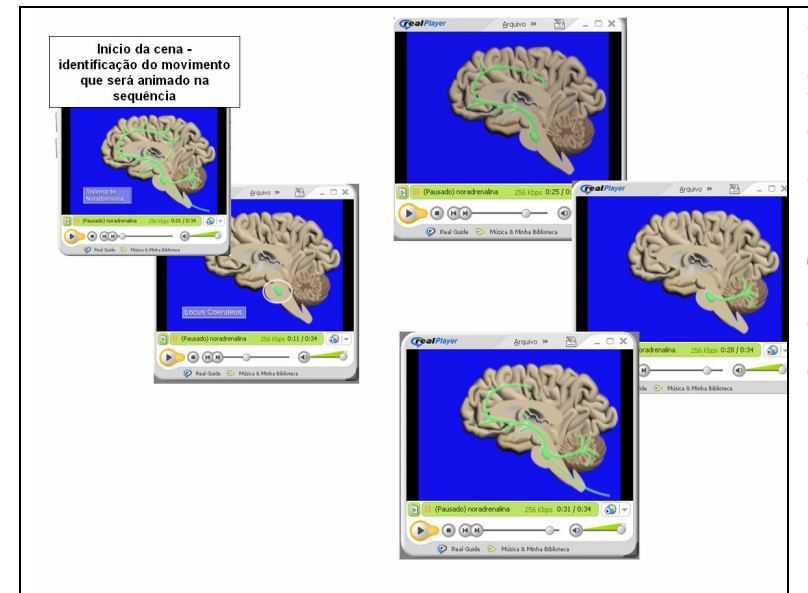

visualizar as vias do neurotransmissor nordrenalina, desde o núcleo de partida, locus ceruleus, e as várias projeções de axônios em direção aos locais-alvo, ou seja, neo-cortex, sistema límbico, cerebelo, hipocampo, etc. Trata-se de uma representação do que ocorre com a corrente elétrica, que percorre esta via e libera o neutransmisor nos locais-alvo.

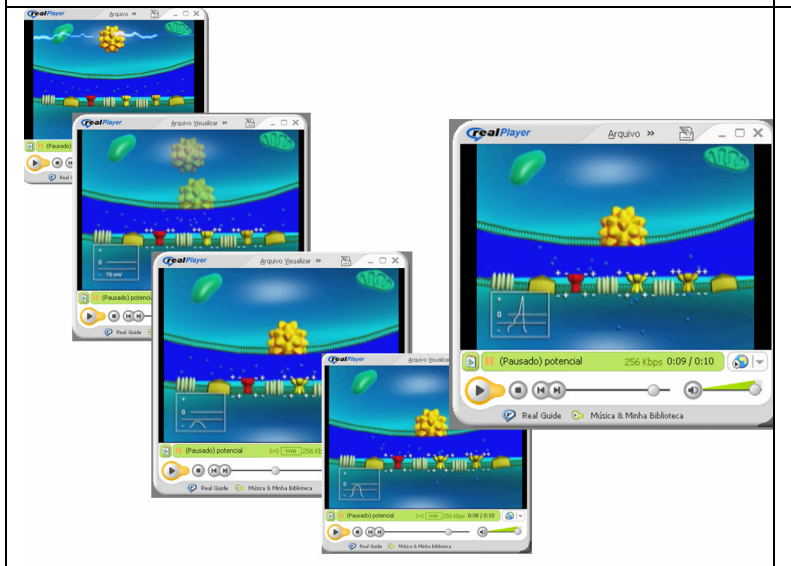

Animação representando em 3D o potencial de ação: a pré-sinapse, com a chegada de um potencial elétrico; a liberação do neutransmissor; seu acoplamento a receptores na parte pós-sináptica e a conseqüente abertura de canais iônicos na formação dos potenciais pós-sinápticos.

Um gráfico, na parte inferior esquerda da tela, acompanha a animação, reproduzindo a formação do potencial, na forma de um sinal elétrico.

A representação busca dar uma visualização dinâmica de um fenômeno que, de outra maneira, poderia ser apenas imaginado pelos alunos.

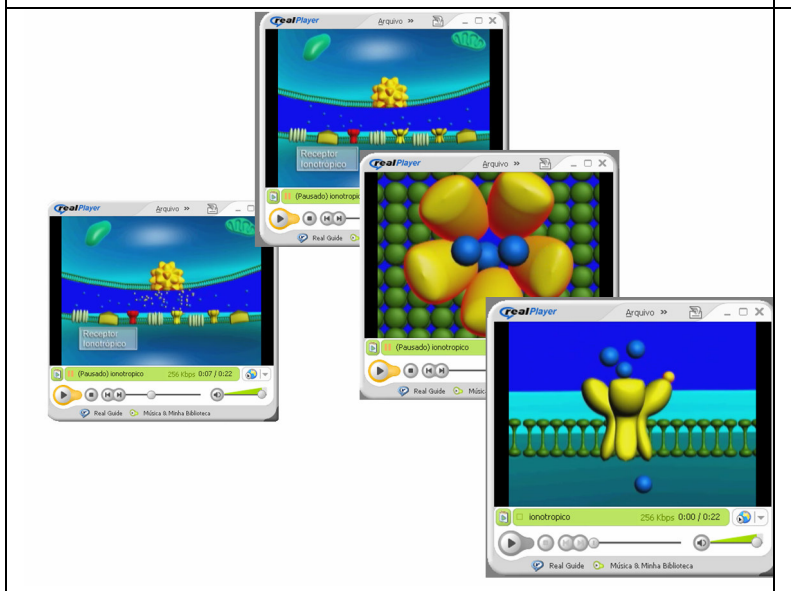

O objetivo desta animação foi facilitar a visualização do que seria uma sinapse ocorrida através da passagem de um potencial de ação através de receptores ionotrópicos. A animação inicia com a chegada do sinal elétrico (primeira tela, à esquerda da figura ao lado), o qual provoca liberação de neurotransmissor contido nas vesículas pré-sinápticas (representadas por pequenas esferas em movimento). $\mathrm{O}$ neurotransmissor liberado vai estimular os receptores pós-sinápticos.

$\mathrm{Na}$ seqüência (tela do alto, na figura), mostrou-se uma pequena abertura no canal e a cena muda, na animação, para uma visão em detalhe desta abertura, como se estivesse sendo vista de cima, em um exemplo da linguagem audiovisual de corte para o detalhe do que estava sendo mostrado. Logo 
em seguida, na última cena da animação, corta-se novamente para uma imagem do que seria o mesmo canal, visto lateralmente, aberto e permitindo a passagem dos íons, representados pelas esferas, agora vistas em tamanho adaptado para o que seria a visão em detalhe, passando pelo canal aberto.

Na tabela 2, serão apresentados exemplos tirados de um vídeo produzido para funcionar como uma aula completa de Embriologia, da professora Adriana Bos-Mikich, do Departamento de Ciências Morfológicas, do Instituto de Ciências Básicas da Saúde (ICBS), da UFRGS.

Tabela 2 - Exemplo de Vídeo Educacional em Embriologia

\begin{tabular}{|l|l|}
\hline Imagens tiradas de cenas dos vídeos & Descrição e análise das imagens \\
\hline & $\begin{array}{l}\text { A filmagem foi feita usando o recurso } \\
\text { chamado chroma key, muito conhecido pelas } \\
\text { apresentações dos meteorologistas, na } \\
\text { televisão. A professora está apresentando o } \\
\text { tema, em estúdio, tendo como cenário um } \\
\text { fundo azul. As imagens (são apresentadas } \\
\text { lâminas, mas poderiam ser qualquer tipo de } \\
\text { imagem, estática ou dinâmica) estão sendo } \\
\text { inseridas eletronicamente, o que viabiliza } \\
\text { tamanho maior e melhor visibilidade dos } \\
\text { detalhes ampliados da imagem. Isso pode ser } \\
\text { visto na primeira e da terceira telas, que } \\
\text { mostram imagens vistas ao microscópio. As } \\
\text { legendas e as caixas de texto foram colocadas } \\
\text { posteriormente, em locais precisos, definidos } \\
\text { pela professora } 4 \text { criando um recursos de } \\
\text { apoio à compreensão e da localização das } \\
\text { áreas, dos detalhes visuais e, na segunda tela, } \\
\text { na identificação das etapas do processo que } \\
\text { está sendo representado na lâmina, onde este } \\
\text { recurso vai surgindo na tela no momento } \\
\text { específico da apresentação. }\end{array}$ \\
\hline
\end{tabular}

${ }^{4} \mathrm{O}$ vídeo foi gravado experimentalmente, com planejamento ainda incipiente, em instalações do Centro Naiconal de Supercomputação (CESUP), na UFRGS, quando encontravam-se em fase de implantação. Sugere-se que, com o domínio da técnica e com o entrosamento adequado com os professores, será possível planejar a inserção desses recursos (legendas, caixas de texto) já na captação das imagens, o que deverá otimizar o ciclo de produção dos vídeos educacionais. 


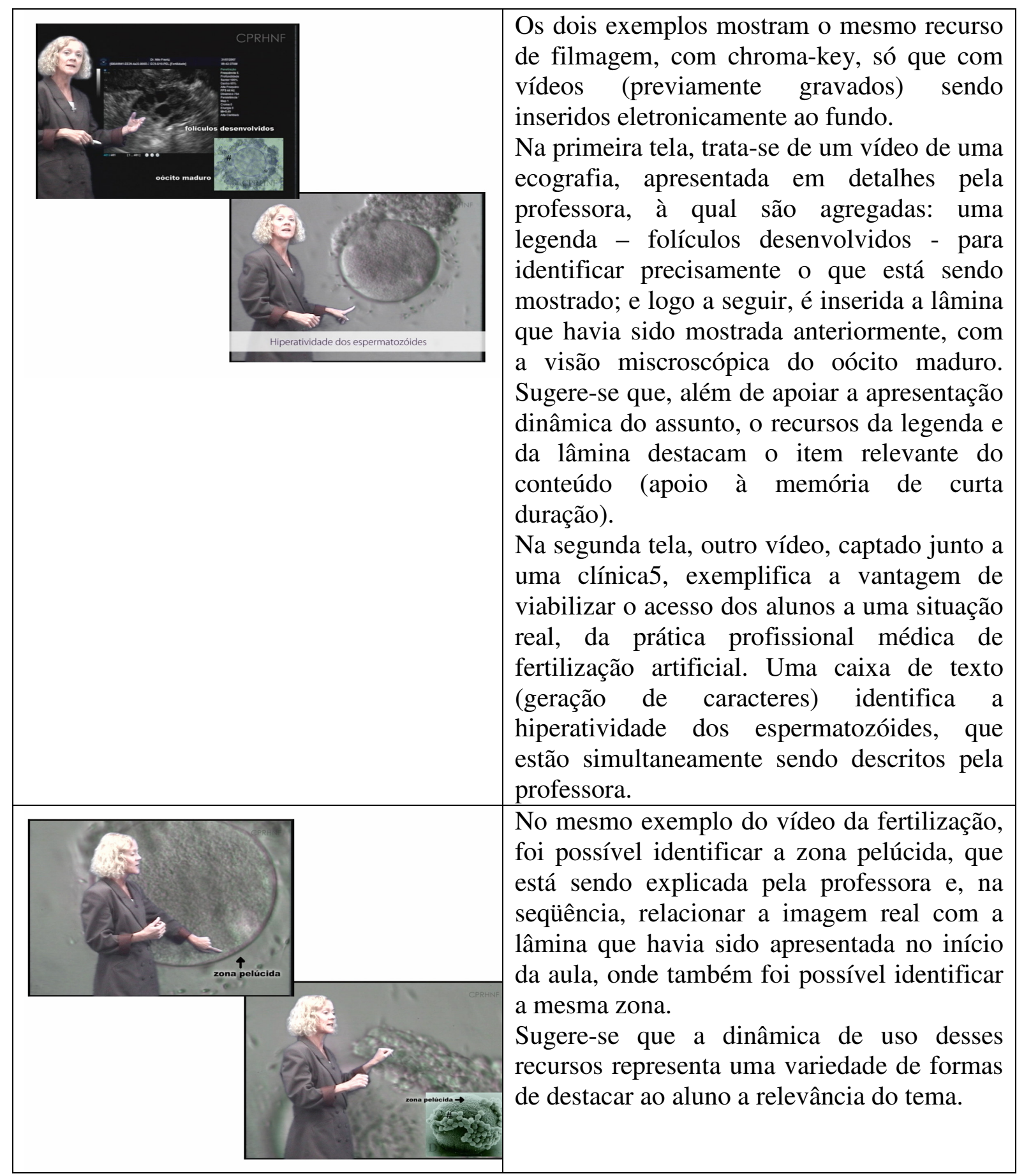

${ }^{5}$ Centro de Pesquisa e Reprodução Humana Nilo Frantz, em Porto Alegre. 


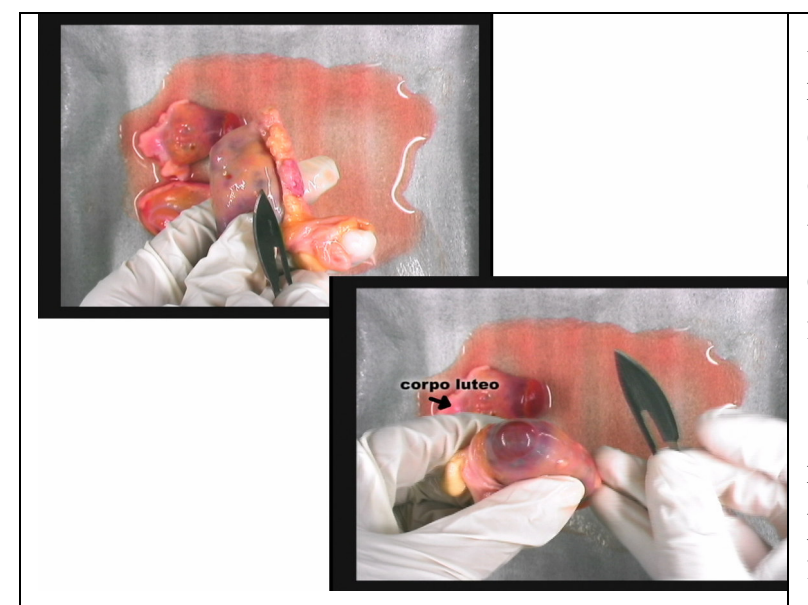

As telas exemplificam a possibilidade de realização de anatomia de um ovário de vaca, com possibilidade de visualização detalhada dos itens que estão sendo apresentados. A título de reflexão sobre a vantagem do vídeo em relação a uma atividade prática dessa natureza, além da questão ética com relação aos animais, imagine-se um grupo de 30 alunos em uma bancada onde estivesse sendo realizada esta anatomia, para se perceber que poucos iriam ter acesso visual detalhado, da forma como podem fazer na tela.

Além disso, a segunda tela mostra o uso das legendas, editadas posteriormente, com a professora, para identificar precisamente o que está sendo mostrado: corpo lúteo.

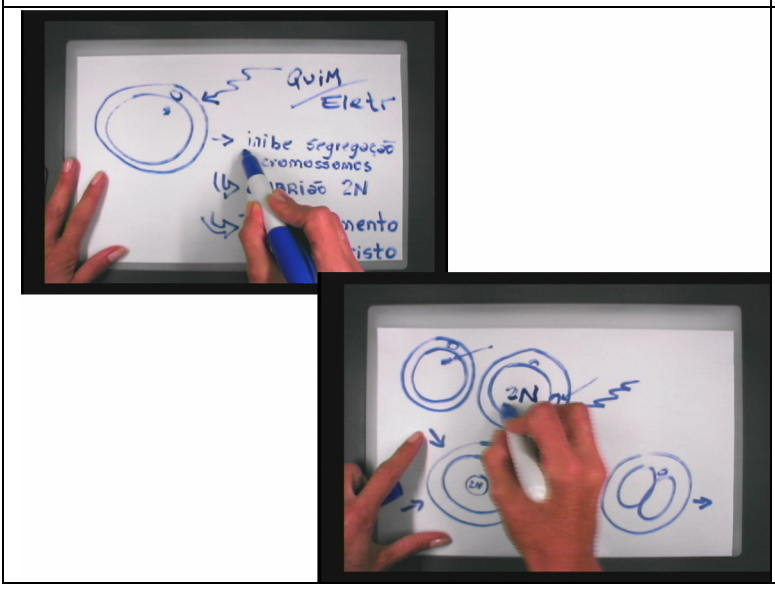

Além das lâminas, dos vídeos e da anatomia, ao vivo, a professora também testou a possibilidade de escrever as informações que julgou necessário, para explicar o tema da fertilização e de ativacao partenogenética para derivação de células-tronco embrionárias.

Ressalte-se a importância de que a escrita precisa ser visível, com boa iluminação e detalhes compreensíveis. Nesse sentido, o velho quadro-negro se renova e também ganha seu espaço na nova tecnologia.

\section{Conclusões}

Procurou-se, neste trabalho, apresentar aos professores do ensino médico, em especial, e a todos os professores, em geral, uma justificativa, baseada em exemplos, para a idéia de que os vídeos educacionais não só são importantes recursos didático-pedagógicos que contribuem para a aprendizagem dos alunos, mas que representam um valor agregado ao trabalho do professor. Por representarem um instrumento de qualificação da atividade docente, porque apóiam de forma criativa e eficiente a representação e a exposição do conhecimento dos professores, os vídeos educacionais devem ser objeto de estudo, pesquisa e profissionalização das equipes de produção, em todas as instituições que pretendem atualizar sua base tecnológica, segundo as necessidades impostas pelo Século XXI. Não foi escopo do artigo descrever estratégias de profissionalização de equipes, ou mesmo apresentar fundamentos técnicos de produção de vídeos. Estes são conhecimentos extensos, consolidados nas áreas profissionais de comunicação social. Buscou-se apontar para a relevância de um enfoque desapaixonado sobre o tema, para que as atividades de produção de vídeos possam fazer parte do planejamento dos gestores educacionais, tanto quanto o são as bibliotecas e editoras, de acordo com as necessidades e possibilidades de cada 
instituição. Em uso individual, na sala de aula, em grupo ou em cursos à distância, os vídeos caracterizam um acervo multimídia indispensável ao novo aprendizado, podendo inclusive aliviar os professores das atividades repetitivas (dar sempre a mesma aula) e, com isso, disponibilizar aos alunos os conteúdos e valorizar sua própria presença, em aula ou em comunicações on-line, para solucionar dúvidas e atualizar informações sobre pesquisas recentes.

Compreender os recursos dos vídeos educacionais e seu potencial, como recurso isolado ou integrado a ambientes multimídia com outras funcionalidades, impõe aos professores conscientizar-se sobre como agregar esse valor à identidade docente. Em especial, aos professores da área médica, cabe contribuir para que não sejam disseminadas crenças sem fundamento biológico a respeito da ineficiência desse tipo de recurso e seu uso na atividade didático-pedagógica - como a de que os vídeos são obsoletos como linguagem educacional - e, ao contrário, explorar seu uso e desenvolver instrumentos científicos e experimentais de observação para validar e melhorar seus processos de produção e uso.

\section{Agradecimentos}

Á bolsista Cláudia Parraga que modelou parte das imagens dos vídeos de Farmacologia. À bolsista Daiana Vivan, que gravou e editou o vídeo de Embriologia.

Ao Centro de Pesquisa e Reprodução Humana Nilo Frantz (Porto Alegre) pelo uso das imagens e vídeos usados pela professora Adriana Bos-Mikich.

\section{Referências}

CABRAL JR, P. A. de F. ; TIMM, M. I.; THADDEU, R. C., LEAL, M. B.; ZARO, M. A.. Produção e Validação de Aula em Vídeo como Objeto de Ensino para o Curso de Medicina. RENOTE - Revista Novas Tecnologias na Educação, V. 3, N2, 2005. Disponível em: http://www.cinted.ufrgs.br/renote/nov2005/artigosrenote/a70_aulavideo.pdf Acesso em 3110-2008.

SCHNAID, F.; ZARO, M.; TIMM, M.I.(org.) Ensino de Engenharia: do positivismo à construção das mudanças para o Século XXI, Ed. da UFRGS, Porto Alegre, 2006.

TIMM, M. I. ; ZARO, M. A. ; SCHNAID, F. ; CABRAL JR, P. A. F. ; THADDEU, R. C. Tecnologia educacional: apoio à representação do professor de Ciência e Tecnologia e instrumento de estudo para o aluno. RENOTE - Revista Novas Tecnologias na Educação, Porto Alegre, v. 2, n. 2, 2004. 